\title{
Large Signal Analysis of a New Meander Line Topology for W-band Traveling Wave Tubes
}

\author{
Juan M. Socuéllamos ${ }^{1}$, Rosa Letizia ${ }^{1}$, Roberto Dionisio ${ }^{2}$ and Claudio Paoloni ${ }^{1}$ \\ ${ }^{1}$ Engineering Department, Lancaster University, Lancaster, United Kingdom \\ ${ }^{2}$ European Space Technology and Research Centre, European Space Agency, Noordwijk, The Netherlands
}

\begin{abstract}
Satellite distribution of high data rate needs wide frequency band. The millimeter waves, in particular the Wband, provide wide bandwidth and relatively low attenuation. The link transmission power can be provided only by Traveling Wave Tubes (TWTs). A new meander line topology to be used as slow wave structure for 71-76 GHz TWTs, with improved performance in comparison to the conventional one, is proposed. The new meander line compared to a standard meander line shows flatter gain and higher output power.
\end{abstract}

Keywords-Traveling wave tube, Slow wave structure, Meander line, W-band

\section{INTRODUCTION}

The allocated W-band (71-76 GHz) for satellite downlink aims to provide a suitable transmission window for high data transfer rate [1]. The wideband transmission power for the link can be only provided by TWTs. The traditional use of helix-type slow wave structures is not realizable at high frequencies due to manufacturing limitations. Different topologies of slow wave structures must be found $[2,3]$.

Meander lines have been studied in detail as slow wave structures for traveling wave tubes due to their favourable properties. This sort of structures is simple to fabricate using microfabrication techniques, with easy assembly and suitable for large scale production [4]. Moreover, meander structures are potentially capable of working with lower operation voltage as well as higher interaction impedance while providing similar performance as three-dimensional full metal structures $[5,6]$.

However, a remarkable drawback of meander lines is the narrow region of synchronism between the electromagnetic wave and the electron beam which limits the effective bandwidth. This issue is greatly influenced by the shape of the metal line and can also be moderately controlled modifying the dimensions of the metal strip as well as the size of the substrate where the metal line is placed. In this paper, a new meander line topology is studied aiming to improve the gain and output power flatness of this kind of structures.

The new structure was previously analysed and compared with a standard meander line of exactly the same dimensions by means of cold simulations. It was found that the dispersion slope of the new meander line was reduced by $32 \%$ while the interaction impedance was increased by $6 \%$ in comparison with the standard line [7]. Now, in this work, both structures are compared for large signal simulations obtaining results for the gain and output power within the frequency range of interest.

\section{RESULTS}

The proposed new topology and the standard meander line are designed using the same dimensions as shown in Fig.
1. The copper line, assumed with reduced conductivity $\sigma=$ $2.25 \times 10^{7} \mathrm{~S} / \mathrm{m}$ to account for the metal loss at W-band [8], is placed on top of an alumina substrate with relative permittivity $\varepsilon_{\mathrm{r}}=9.9$. Both the cold and hot simulations have been performed using CST Microwave Studio [9].

The dispersion curves of the new and standard meander lines are compared in Fig. 2. The dispersion of the standard line presents a more pronounced slope than the one of the new line, so it is to be expected that the new meander will be benefited from a flatter gain and output power within the 71$76 \mathrm{GHz}$ range. In order to approximately obtain the operational voltage that will be used for the large signal simulations, different beam lines are plotted along the dispersion curves. The $4.4 \mathrm{kV}$ beam line is the one that better matches the dispersion relation of the new meander so that the maximum output is centered within the frequency range of interest. As the standard structure presents a different dispersion curve, the suitable beam line for that structure has also a different value. For this case, the selected voltage is $3.2 \mathrm{kV}$ as shown in Fig. 2.

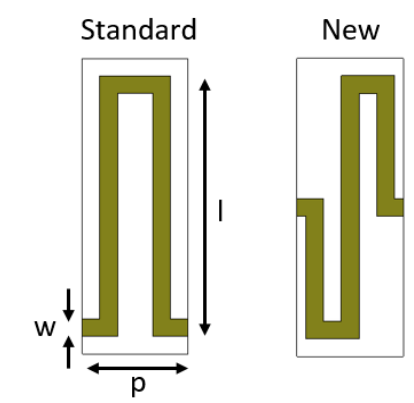

\begin{tabular}{|c|c|}
\hline Parameter & Value $(\boldsymbol{\mu m})$ \\
\hline I & 300 \\
\hline p & 120 \\
\hline t & 3 \\
\hline w & 20 \\
\hline
\end{tabular}

Fig. 1. Shape of the standard and new meander lines as well as the dimensions of both structures. The metal strips are designed with length $l$, thickness $t$ and width $w$. The period of the structure is denoted with $p$.

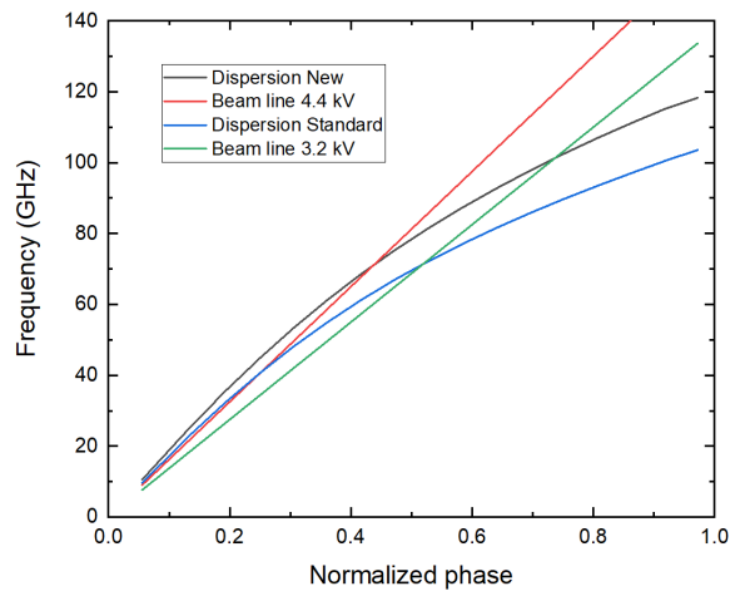

Fig. 2. Dispersion curve for the new (black curve) and standard (blue curve) meander lines as well as their corresponding beam lines of $4.4 \mathrm{kV}$ (red line) and $3.2 \mathrm{kV}$ (green line) 
Once the beam voltage has been determined, the large signal performance of the new structure can be analysed. The new meander and the standard line are compared using the same hot parameters.

A cylindrical electron beam with a radius of $80 \mu \mathrm{m}$ is placed at a distance of $25 \mu \mathrm{m}$ from the metal strip. The current of the electron beam is $50 \mathrm{~mA}$, equivalent to a current density of $250 \mathrm{~A} / \mathrm{cm}^{2}$. A radiofrequency signal with $4 \mathrm{~mW}$ input power is introduced into the structure by the input ports. The waveguides and the meander line are connected following an E-field probe coupling transition [8], which was shown to provide adequate transmission and low losses when applied to the new meander line [7]. Finally, a magnetic field of 0.6 $\mathrm{T}$ is selected to focus the electron beam. The only difference between both simulations is the value of the voltage as shown in Fig. 2.

In order to analyse the large signal performance of the structure, both the gain and output power of the new meander are compared with the results for the standard line. The gain for both structures within the 71-76 GHz frequency range is represented in Fig. 3. The new design presents a lower variation between the different points in the curve. Moreover, the gain for the standard line drops dramatically as the frequency approaches the edges of the band. The maximum gain for the new design is $23.8 \mathrm{~dB}$, a higher value in comparison with $22.8 \mathrm{~dB}$ of the standard line.

Similarly, Fig. 4 shows the comparison of the output power between both structures within the bandwidth of interest. The output power presents a smaller variation among the different frequencies for the case of the new meander line. The maximum output power of the new topology is $1 \mathrm{~W}$, an increased value compared to the $0.8 \mathrm{~W}$ that the standard line provides.

The low levels of output power can be explained considering the low input power and current used for the simulations. Moreover, only a single section is analysed. The complete structure can be designed to have two or more sections in order to increase the gain and meet the requirements of traveling wave tubes for space applications.

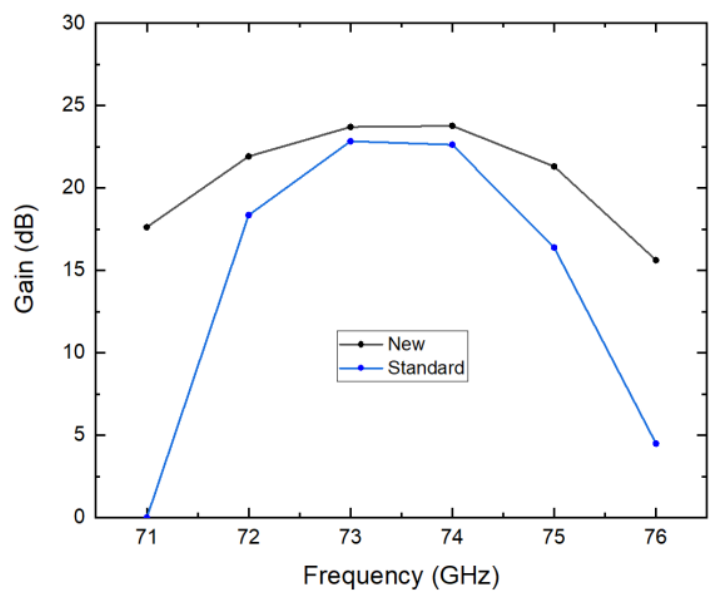

Fig. 3. Gain of the new (black line) and standard (blue line) meanders from 71 to $76 \mathrm{GHz}$.

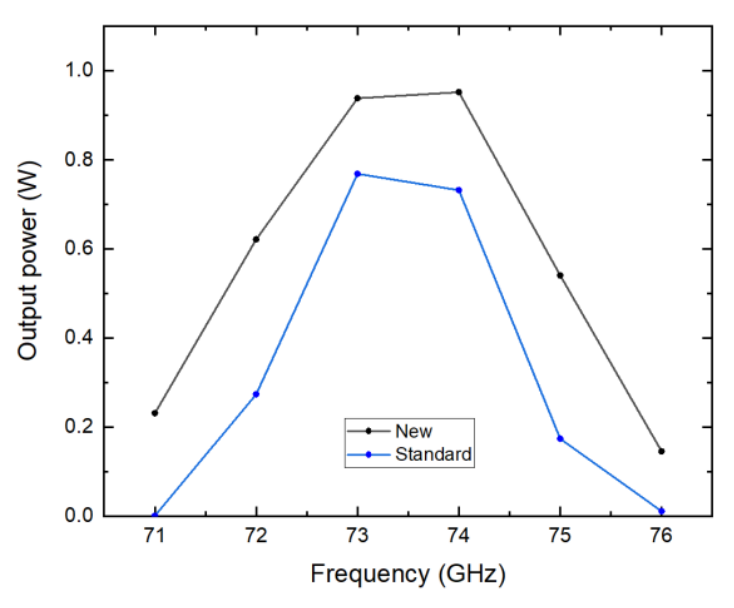

Fig. 4. Output power of the new (black line) and standard (blue line) meanders from 71 to $76 \mathrm{GHz}$.

\section{CONCLUSION}

A new meander line topology has been proposed to improve the gain and output power flatness of this kind of slow wave structures. Cold results revealed a gentler dispersion curve and higher interaction impedance in comparison with the standard meander line shape. These results have been transferred to the large signal analysis of the structure showing that the new design offers flatter gain and higher output power within the $71-76 \mathrm{GHz}$ frequency range. Further work will be focus on improving the output power and gain of the structure.

\section{REFERENCES}

[1] N. Ayllon, P. Angeletti, M. Ludwig and R. Dionisio, "An overview of European Spaceborne Vacuum Tube Amplifiers and System Needs", in Proc. IEEE 18 ${ }^{\text {th }}$ Int. Vac. Electron. Conf., London, UK, April 2017.

[2] M. Mineo and C. Paoloni, "Double Corrugation Rectangular Waveguide Slow-wave Structure for THz Vacuum Devices", IEEE Trans. on Electron Devices, vol. 57, no. 11, pp.3169-3175, November 2010

[3] X. Li, X. Huang, S. Mathisen, R. Letizia, C. Paoloni, "Desing of 71$76 \mathrm{GHz}$ Double-Corrugated Waveguide Traveling-Wave Tube for Satellite Downlink", IEEE Trans. on Electron Devices. vol. 65, no. 6 , pp. 2195-2200, June 2018.

[4] S. Wang, S. Aditya, X. Xia, Z. Ali and J. Miao, "On-Wafer Microstrip Meander Line Slow Wave Structure at Ka-Band", IEEE Trans. on Electron Devices. vol. 65, no. 6, pp. 2142-2148, June 2018.

[5] F. Shen, Y. Wei, H. Yin, Y. Gong, X. Xu, S. Wang, W. Wang and J. Feng, "A Novel V-shaped Microstrip Meander Line Slow Wave Structure for W-band MMPM", IEEE Trans. on Plasma Sci., vol. 40, no. 2, pp. 463-469, February 2012.

[6] W. Zhang, Y. Wang, B. Qu, "Design of Folded Double-Ridged Waveguide Slow-Wave Structure", in Proc. $19^{\text {th }}$ Int. Vac. Electron. Conf., Monterey, USA, April 2018.

[7] J. M. Socuéllamos, R. Letizia, R. Dionisio, C. Paoloni, "Preliminary Study of a New Meander Line for W-band TWT", in Proc. $20^{\text {th }}$ Int. Vac. Electron. Conf., Busan, South Korea, April 2019.

[8] C. Kory, R. L. Ives, M. Read, J. Booske, H. Jiang, D. van der Weide, S-J Ho, S. Sengele and P. Phillips "Overview of W-band Travelling Wave Tube Programs", in Proc. $7^{\text {th }}$ Int. Vac. Electron. Conf., Monterey, USA, April. 2006.

[9] CST-MWS. [Online]. Available: https://www.cst.com/products/csts2 